\title{
Osteonecrose em Pacientes com Lúpus Eritematoso Sistêmico ${ }^{(*)}$
}

\section{Osteonecrosis in Systemic Lupus Erythematosus Patients}

\author{
Elaine Marcelina Claudio Sella ${ }^{(1)}$, Maria Rosenilda Petronila de Carvalho ${ }^{(2)}$, Emilia Inoue Sato ${ }^{(3)}$
}

\section{RESUMO}

Osteonecrose é uma complicação relativamente comum em pacientes com lúpus eritematoso sistêmico (LES). Objetivo: avaliação dos possíveis fatores de risco associados à presença de osteonecrose em pacientes com LES. Métodos: Foram incluídos neste estudo pacientes com LES [critérios para a classificação do American College of Rheumatology (ACR)] que apresentaram osteonecrose durante a evolução da doença. O grupo controle foi constituído por pacientes com LES sem indícios de osteonecrose. O diagnóstico de osteonecrose foi confirmado por radiografia simples, cintilografia óssea e/ou ressonância nuclear magnética. Resultados: quatorze pacientes com LES desenvolveram osteonecrose (10 mulheres e 4 homens, $64 \%$ de cor branca, $33 \pm 13$ anos e $120 \pm 67$ meses de LES). O grupo controle, pareado por sexo e tempo de diagnóstico de LES, foi constituído por pacientes com LES e sem osteonecrose ( $\mathrm{n}=14,57 \%$ de cor branca, $33 \pm 7$ anos e $111 \pm 54$ meses de LES). O escore do systemic lupus international collaborating clinics/ACR damage index for systemic lupus erythematosus (SLICC/ACR-DI) foi maior nos pacientes com

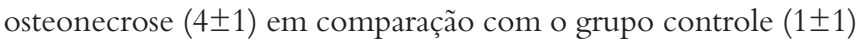
$[\mathrm{p}<0,001]$. Pacientes com osteonecrose apresentaram maior número de outros danos musculoesqueléticos permanentes quando comparados com os pacientes controles $(29 \%$ versus zero, respectivamente; $p=0,034)$. A vasculite digital foi a variável associada à osteonecrose $(\mathrm{p}=0,021)$. Não houve associação significante entre tempo de uso ou dose cumulativa de prednisona e osteonecrose nos pacientes regularmente acompanhados com o diagnóstico de LES ( $p=0,624$ e $p=0,806$, respectivamente). Conclusões: História prévia de vasculite digital foi fator de risco para o desenvolvimento de osteonecrose. O risco de pacientes com vasculite digital pregressa apresentar osteonecrose foi nove vezes maior que o de pacientes com LES sem vasculite prévia.

Palavras-chave: lúpus eritematoso sistêmico, osteonecrose, fatores de risco, vasculite.

\begin{abstract}
Osteonecrosis is a relatively common complication in systemic lupus erythematosus (SLE) patients. Objective: To evaluate the possible risk factors associated with osteonecrosis in SLE patients. Methods: SLE patients [according to American College of Rheumatology (ACR) criteria] who presented osteonecrosis were included in this study. SLE patients with no symptomatic osteonecrosis constituted the control group. Osteonecrosis was confirmed by radiographic evaluation, bone scintigraphy and/or magnetic resonance imaging. Results: The study group was constituted by 14 SLE patients with osteonecrosis (10 women and 4 men, $64 \%$ of them white, $33 \pm 13$ years old and $120 \pm 67$ months of disease duration). Sex and time of SLE diagnosis matched patients without osteonecrosis constituted the control group ( $n=14,57 \%$ of them white, $33 \pm 7$ years old and $111 \pm 54$ months of SLE). Systemic lupus international collaborating clinics/ACR damage index for SLE (SLICC/ACR-DI) score was higher in patients with osteonecrosis (4 \pm 1$)$ compared with control group (1 \pm 1$)[p<0.001]$. Patients with osteonecrosis had higher number of other musculoskeletal irreversible damage when compared with the control group (29\% versus 0, respectively; $p=0.034)$. Digital vasculitis was the variable associated with osteonecrosis $(p=0.021)$. There was no significant association between duration of prednisone use or prednisone cumulative dose and osteonecrosis in patients with regular follow-up at the Institution ( $p=0.624$ and $p=0.806$, respectively). Conclusions: Previous history of digital vasculitis was a risk factor for the development of osteonecrosis. Patients with digital vasculitis history had 9 times more risk to present osteonecrosis than patients without previous vasculitis
\end{abstract}

Keywords: systemic lupus erythematosus, osteonecrosis, risk factors, vasculitis.

\footnotetext{
* Trabalho realizado na Disciplina de Reumatologia da Universidade Federal de São Paulo/Escola Paulista de Medicina (Unifesp/EPM), São Paulo-SP, Brasil Recebido em 4/10/2004. Aprovado, após revisão, em 10/01/2005.

1. Mestre em Reumatologia. Disciplina de Reumatologia da Unifesp/EPM, São Paulo-SP, Brasil

2. Doutora em Fisioterapia. Disciplina de Reumatologia da Unifesp/EPM, São Paulo-SP, Brasil.

3. Professora Titular. Disciplina de Reumatologia da Unifesp/EPM, São Paulo-SP, Brasil.

Endereço para correspondência: Dra. Elaine Marcelina Claudio Sella. Universidade Federal de São Paulo/Escola Paulista de Medicina (Unifesp/EPM). Disciplina de Reumatologia. Rua Botucatu, 740, $3^{\circ}$ andar, Vila Clementino, CEP 04023-062, São Paulo, SP, Brasil. E-mail: emiliasato@reumato.epm.br
} 


\section{INTRODUÇÃO}

Osteonecrose, necrose óssea avascular, isquêmica ou asséptica pode ser definida como a morte óssea que resulta no colapso da arquitetura óssea, levando à dor articular, destruição óssea e perda de função. As causas da osteonecrose incluem a interrupção do suprimento vascular resultante de trauma local ou condições sistêmicas não traumáticas. Dentre as condições não traumáticas citam-se doenças hematológicas, metabólicas/endocrinológicas, gastrintestinais, neoplásicas, infecciosas, reumatológicas, ortopédicas, além de fatores ambientais e iatrogênicos ${ }^{(1,2)}$.

Condições associadas à osteonecrose incluem: administração de corticosteróides, alcoolismo, infecções, eventos hiperbáricos, defeitos de coagulação, doenças infiltrativas de medula óssea e desordens auto-imunes. Nos casos de trauma, a interrupção do fluxo sangüíneo para o osso propicia a necrose, e, em condições não traumáticas, o mecanismo patogenético não é totalmente conhecido ${ }^{(2)}$.

A definida associação entre osteonecrose e lúpus eritematoso sistêmico (LES) foi inicialmente documentada por Dubois e Cozen, em 1960. Os autores relataram 11 casos de osteonecrose $(2,8 \%)$, numa série de 400 pacientes com $\mathrm{LES}^{(3)}$. Posteriormente, osteonecrose foi referida em freqüências que variavam de $11 \%$ a $30 \%$ dos pacientes com LES em estudos internacionais ${ }^{(4-9)}$. Maior prevalência de osteonecrose foi descrita em trabalhos utilizando-se a ressonância nuclear magnética ${ }^{(10,11)}$.

Osteonecrose é um dos itens pontuados na avaliação de danos permanentes não reversíveis em pacientes com LES [systemic lupus international collaborating clinics/American College of R heumatology damage index for systemic lupus erythematosus (SLICC/ACR-DI) ${ }^{(12,13)}$. Em alguns estudos, o quadril foi a articulação mais acometida pela osteonecrose, seguida pelos joelhos e ombros ${ }^{(14,15)}$. A cabeça femoral foi o local mais afetado ${ }^{(8,16,17)}$.

Diferentes fatores de risco associados ao desenvolvimento de osteonecrose foram identificados em pacientes com LES, como presença do fenômeno de Raynaud ${ }^{(14)}$, doença neurológica ${ }^{(8)}$, vasculite ${ }^{(7)}$, anticorpos antifosfolípides ${ }^{(7,8)}$ e anormalidades de coagulação ${ }^{(18,19)}$.

Acredita-se, ainda, que os corticosteróides relacionemse positivamente com o aparecimento da osteonecrose $\mathrm{e}^{(7-9,11)}$. Porém, não há consenso sobre a duração da corticoterapia, a via de administração ou as doses máxima e/ou cumulativa de esteróides implicadas no risco de osteonecrose.

O objetivo principal deste estudo foi avaliar os possíveis fatores de risco associados com a presença de osteonecrose em pacientes com LES, atendidos em um serviço terciário de saúde.

\section{PACIENTES E MÉTODOS}

No período compreendido entre janeiro e setembro de 2003 foram revisados 130 prontuários médicos dos pacientes com diagnóstico de LES em seguimento no Ambulatório de Doenças do Tecido Conjuntivo, da Disciplina de Reumatologia do Hospital São Paulo - Universidade Federal de São Paulo/Escola Paulista de Medicina (Unifesp/EPM).

Foram incluídos neste estudo somente pacientes com o mínimo de quatro critérios para a classificação de LES de acordo com o ACR ${ }^{(20,21)}$ e que apresentaram osteonecrose durante a evolução da doença. O grupo controle foi constituído por pacientes com LES sem osteonecrose. O projeto de pesquisa foi previamente aprovado pela Comissão de Ética em Pesquisa Médica da Instituição.

A revisão dos prontuários foi realizada com o intuito de se obter informações a respeito dos dados demográficos, tempo de diagnóstico do LES, critérios diagnósticos e outras manifestações clínicas da doença, dados laboratoriais gerais e imunológicos e escores de SLICC/ACR-DI. Para o cálculo do tempo de uso e dose cumulativa de prednisona foram considerados apenas os pacientes que tiveram diagnóstico de LES na instituição e permaneceram, desde então, em acompanhamento médico regular.

O diagnóstico clínico de osteonecrose foi confirmado por radiografia simples, cintilografia óssea e/ou ressonância nuclear magnética.

Análise estatística: o teste exato de Fisher foi utilizado para verificar a relação de dependência entre as variáveis categóricas. O teste de Mann-Whitney foi usado para comparar os subgrupos de pacientes com e sem osteonecrose com relação às variáveis quantitativas ${ }^{(22)}$. Para os testes acima descritos foram considerados resultados estatisticamente significantes aqueles com valores do nível descritivo $(\mathrm{p})<0,05$, os quais foram assinalados com um asterisco $\left(^{*}\right)$.

\section{RESULTADOS}

\section{DADOS DEMOGRÁFICOS}

Durante o período estudado foram identificados $14 \mathrm{pa}-$ cientes com LES que desenvolveram osteonecrose $(10 \mathrm{mu}-$ lheres e 4 homens, $64 \%$ de cor branca, $33 \pm 13$ anos e $120 \pm 67$ meses de LES). O grupo controle $(\mathrm{n}=14)$, pareado por sexo e tempo de diagnóstico de LES, foi constituído por pacientes com LES e sem osteonecrose $(57 \%$ de cor branca, $33 \pm 7$ anos e 111 \pm 54 meses de LES) (Tabela 1). 
TABELA 1

Medidas de TENDÊNCIA CENTRAL E DE DisPERSÃo DE IDADE ATUAL, TEMPO DE DIAGNÓSTICO DE LES, IDADE À ÉPOCA DO DIAGNÓSTICO, NÚMERO DE CRITÉRIOS DO ACR E ESCORES DO SLICC/ACR-DI DOS 28 PACIENTES

\begin{tabular}{|c|c|c|c|c|}
\hline Variáveis & 28 LES & $\begin{array}{c}14 \text { LES } \\
\text { Osteonecrose }\end{array}$ & $\begin{array}{c}14 \text { LES } \\
\text { Controle }\end{array}$ & $\mathrm{p}$ \\
\hline \multicolumn{5}{|l|}{$\begin{array}{l}\text { Idade Atual } \\
\text { (anos) }\end{array}$} \\
\hline $\mathrm{MD} \pm \mathrm{DP}$ & $33 \pm 10$ & $33 \pm 13$ & $33 \pm 7$ & \\
\hline Mediana & 31 & 29 & 32 & 0,505 \\
\hline Mín./máx. & $20 / 67$ & $20 / 67$ & $22 / 44$ & \\
\hline \multicolumn{5}{|c|}{$\begin{array}{l}\text { Tempo do LES } \\
\text { (meses) }\end{array}$} \\
\hline $\mathrm{MD} \pm \mathrm{DP}$ & $116 \pm 60$ & $120 \pm 67$ & $111 \pm 54$ & \\
\hline Mediana & 99 & 106 & 99 & 0,783 \\
\hline Mín./ máx. & $36 / 259$ & $36 / 259$ & $36 / 227$ & \\
\hline \multicolumn{5}{|c|}{$\begin{array}{l}\text { Idade de início } \\
\text { do LES (anos) }\end{array}$} \\
\hline $\mathrm{MD} \pm \mathrm{DP}$ & $23 \pm 7$ & $23 \pm 8$ & $23 \pm 7$ & \\
\hline Mediana & 21 & 20 & 23 & 0,645 \\
\hline Mín./ máx. & $12 / 46$ & $15 / 46$ & $12 / 37$ & \\
\hline \multicolumn{5}{|l|}{ N ACR } \\
\hline $\mathrm{MD} \pm \mathrm{DP}$ & $7 \pm 1$ & $7 \pm 1$ & $7 \pm 1$ & \\
\hline Mediana & 7 & 7 & 6 & 0,394 \\
\hline Mín./ máx. & $4 / 9$ & $4 / 9$ & $5 / 9$ & \\
\hline \multicolumn{5}{|c|}{ N SLICC/ACR-DI } \\
\hline$M D \pm D P$ & $2 \pm 2$ & $4 \pm 1$ & $1 \pm 1$ & \\
\hline Mediana & 3 & 4 & 1 & $<0,001$ * \\
\hline Mín./ máx. & $0 / 5$ & $2 / 5$ & $0 / 4$ & \\
\hline
\end{tabular}

\section{AVALIAÇÃo DA OSTEONECROSE}

A idade dos pacientes à época da osteonecrose foi $29 \pm 10$ anos (mediana 26 anos; idade mínima de 20 e máxima de 51 anos) com intervalo de tempo entre o diagnóstico de LES e o primeiro evento de osteonecrose de $75 \pm 53$ meses (mediana 58 meses e variação de 24 a 228 meses).

O número total de sítios de osteonecrose foi 34 (variação de 1 a 6 por paciente). Do total de pacientes, 57\% apresentaram um único evento ósseo isquêmico e 13 (93\%) pacientes apresentaram osteonecrose em cabeças femorais (Tabela 2).
TABELA 2

Sítios de OSTEONECROSE NOS 14 PACIENTES COM LES

\begin{tabular}{lcc}
\hline Sítios de osteonecrose & $\begin{array}{c}\text { Unilateral } \\
\text { N pacientes }\end{array}$ & $\begin{array}{c}\text { Bilateral } \\
\text { N pacientes }\end{array}$ \\
\hline Quadril & 3 & 10 \\
Joelho & 1 & 2 \\
Úmero & 0 & 2 \\
Tíbia & 0 & 1 \\
\hline
\end{tabular}

\section{FATORES DE RISCO ASSOCIADOS À OSTEONECROSE}

Não houve diferença estatisticamente significante com relação à idade atual, tempo de LES, idade à época do diagnóstico da doença ou número de critérios do ACR entre pacientes com osteonecrose e grupo controle. Observamos maior escore do SLICC/ACR-DI $(\mathrm{p}<0,001)$ nos pacientes com osteonecrose ( $4 \pm 1$; mediana 4$)$ em comparação com os pacientes controles $(1 \pm 1$; mediana 1$)$ (Tabela 1).

Excluindo-se a pontuação atribuída à osteonecrose, a análise estatística não revelou diferença significante nos escores do SLICC/ACR-DI entre os grupos de pacientes considerados $(2 \pm 1$ e mediana 2 nos pacientes com osteonecrose, e, $1 \pm 1$ e mediana 1 nos pacientes do grupo controle; $\mathrm{p}=0,097)$.

Os danos permanentes em órgãos e/ou sistemas avaliados pelo SLICC/ACR-DI nos dois grupos de pacientes são apresentados na Tabela 3. O grupo de pacientes com osteonecrose apresentou maior número de outros danos musculoesqueléticos permanentes quando comparado com os pacientes controles $(29 \%$ versus 0 respectivamente; $\mathrm{p}=0,034)$.

A vasculite digital pregressa, definida pela impressão clínica do médico observador, foi descrita em número significantemente maior de pacientes com osteonecrose, quando comparado ao grupo controle [11 (79\%) versus 4 (29\%); $\mathrm{p}=0,021]$. A análise estatística epidemiológica mostrou vasculite digital como importante variável associada à osteonecrose $[\mathrm{p}=0,012$, odds ratio $(\mathrm{OR})=9,17$ e intervalo de confiança $(\mathrm{IC})=1,63-51,43]$.

Não houve associação significante entre outras manifestações vasculares, dermatológicas, articulares, de serosas, renais, neurológicas, hematológicas ou imunológicas observadas ao longo da evolução da doença e presença de osteonecrose. As freqüências e porcentagens dos critérios de classificação do ACR e outras manifestações clínicolaboratoriais são mostradas na Tabela 4. 
TABELA 3

FREQÜÊNCIAS E PORCENTAGENS DE PACIENTES COM DANOS PERMANENTES NOS GRUPOS DE PACIENTES COM OSTEONECROSE E CONTROLE

\begin{tabular}{|c|c|c|}
\hline Variáveis SLICC/ACR-DI & $\begin{array}{c}14 \text { LES } \\
\text { Osteonecrose } \\
\mathrm{N}(\%)\end{array}$ & $\begin{array}{c}14 \text { LES } \\
\text { Controle } \\
\mathrm{N}(\%)\end{array}$ \\
\hline Ocular & $1(7 \%)$ & 0 \\
\hline Catarata & 1 & 0 \\
\hline Neuropsiquiátrico & $3(21 \%)$ & $4(29 \%)$ \\
\hline Disfunção cognitiva & 0 & 3 \\
\hline Anticonvulsivantes $>6$ meses & 3 & 0 \\
\hline Acidente vascular cerebral & 0 & 1 \\
\hline Neuropatia craniana & 0 & 1 \\
\hline Renal & $4(29 \%)$ & $4(29 \%)$ \\
\hline Taxa de filtração glomerular reduzida & 0 & 2 \\
\hline Proteinúria $\geq 3,5 \mathrm{~g} / 24$ horas & 4 & 1 \\
\hline Insuficiência renal terminal - diálise & 0 & 1 \\
\hline Pulmonar & $1(7 \%)$ & 0 \\
\hline Fibrose pulmonar & 1 & 0 \\
\hline Cardiovascular & $2(14 \%)$ & 0 \\
\hline Infarto agudo do miocárdio & 1 & 0 \\
\hline Miocardiopatia & 2 & 0 \\
\hline Pericardite por 6 meses & 1 & 0 \\
\hline Vascular periférico & $4(29 \%)$ & $1(7 \%)$ \\
\hline Perda de polpa tecidual & 3 & 1 \\
\hline Estase / trombose venosa & 1 & 0 \\
\hline Gastrintestinal & 0 & 0 \\
\hline Musculoesquelético & $4(29 \%)$ & 0 \\
\hline Osteoporose com colapso vertebral & 1 & 0 \\
\hline Deformidade articular redutível & 1 & 0 \\
\hline Deformidade articular erosiva & 2 & 0 \\
\hline Dermatológico & $4(29 \%)$ & $2(14 \%)$ \\
\hline Alopecia crônica cicatricial & 1 & 1 \\
\hline Cicatriz cutânea extensa & 0 & 2 \\
\hline Ulceração cutânea por mais de 6 meses & 3 & 0 \\
\hline Falência gonadal prematura & $1(7 \%)$ & $1(7 \%)$ \\
\hline Malignidade & 0 & $1(7 \%)$ \\
\hline Metaplasia epidermóide (mucosa jugal) & 0 & 1 \\
\hline
\end{tabular}

SLICC/ACR-DI: Systemic lupus international collaborating clinics / ACR damage index for systemic lupus erythematosus.
TABELA 4

FREQÜÊNCIAS E PORCENTAGENS DAS DIFERENTES MANIFESTAÇÕES CLÍNICO-LABORATORIAIS DOS PACIENTES DO ESTUDO

\begin{tabular}{|c|c|c|c|}
\hline Variáveis & $\begin{array}{c}14 \text { LES } \\
\text { Osteonecrose } \\
\mathrm{N}(\%)\end{array}$ & $\begin{array}{c}14 \text { LES } \\
\text { Controle } \\
\mathrm{N}(\%)\end{array}$ & $\mathbf{P}$ \\
\hline Eritema malar & $11(79 \%)$ & $13(93 \%)$ & 0,596 \\
\hline Lesão discóide & $2(14 \%)$ & $3(21 \%)$ & 1 \\
\hline Fotossensibilidade & $13(93 \%)$ & $14(100 \%)$ & 1 \\
\hline Vasculite de mucosa & $5(36 \%)$ & $6(43 \%)$ & 1 \\
\hline Acometimento articular & $11(79 \%)$ & $11(79 \%)$ & 1 \\
\hline Serosite & $6(43 \%)$ & $4(29 \%)$ & 0,695 \\
\hline Acometimento renal & $8(57 \%)$ & $9(64 \%)$ & 1 \\
\hline Acometimento neurológico (ACR) & $3(21 \%)$ & $1(7,1 \%)$ & 0,596 \\
\hline Acometimento hematológico & $13(93 \%)$ & $10(71 \%)$ & 0,326 \\
\hline AAN & $14(100 \%)$ & $14(100 \%)$ & 1 \\
\hline Anti-dsDNA & $10(71 \%)$ & $6(43 \%)$ & 0,252 \\
\hline Anti-Sm & $6(43 \%)$ & $2(14 \%)$ & 0,209 \\
\hline Anti-SSA/Ro & $6(43 \%)$ & $8(57 \%)$ & 0,706 \\
\hline Anti-SSB/La & $1(7,1 \%)$ & $1(7,1 \%)$ & 1 \\
\hline Anti-RNP & $6(43 \%)$ & $3(21 \%)$ & 0,420 \\
\hline $\mathrm{aCL}$ & $6(43 \%)$ & $5(36 \%)$ & 1 \\
\hline Raynaud & $8(57 \%)$ & $7(50 \%)$ & 1 \\
\hline Vasculite digital & $11(79 \%)$ & $4(29 \%)$ & $0,021^{*}$ \\
\hline Vasculite de grandes vasos & $5(36 \%)$ & $2(14 \%)$ & 0,385 \\
\hline Trombose venosa & $2(4 \%)$ & $1(7,1 \%)$ & 1 \\
\hline SAAF & $2(14 \%)$ & $1(7,1 \%)$ & 1 \\
\hline
\end{tabular}

Teste de Fisher: ${ }^{*} \mathrm{p}<0,05$ (significante); AAN: anticorpos antinucleares; ACL: anticorpos anticardiolipina; ACR: American College of Rheumatology; $\mathrm{p}$ : nível descritivo; SAAF: síndrome de anticorpos antifosfolípides

Nove pacientes (4 com osteonecrose e 5 do grupo controle) tiveram seguimento clínico contínuo em nosso serviço. Não houve associação significante entre maior tempo de uso de prednisona (em meses) ou dose cumulativa de prednisona (em gramas) e presença de osteonecrose $(\mathrm{p}=0,624$ e $\mathrm{p}=0,806$, respectivamente) (Tabela 5). 
TABELA 5

MEdidAs de TENDÊNCIA CENTRAL E DE DISPERSÃO DO TEMPO DE USO E DOSE CUMULATIVA APROXIMADA DE PREDNISONA DOS NOVE PACIENTES COM LES EM SEGUIMENTO CONTÍNUO DESDE O DIAGNÓSTICO DA DOENÇA

\begin{tabular}{|c|c|c|c|c|}
\hline Variáveis & 9 LES & $\begin{array}{c}4 \text { LES } \\
\text { 0steonecrose }\end{array}$ & $\begin{array}{c}5 \text { LES } \\
\text { Controle }\end{array}$ & $\mathrm{p}$ \\
\hline \multicolumn{5}{|c|}{ Corticoterapia (meses) } \\
\hline$M D \pm D P$ & $83 \pm 43$ & $90 \pm 53$ & $77 \pm 38$ & \\
\hline Mediana & 95 & 83 & 95 & 0,624 \\
\hline Mín./máx. & $22 / 153$ & $43 / 153$ & $22 / 110$ & \\
\hline \multicolumn{5}{|c|}{ Dose cumulativa (g) } \\
\hline $\mathrm{MD} \pm \mathrm{DP}$ & $53 \pm 26$ & $58 \pm 26$ & $49 \pm 29$ & \\
\hline Mediana & 42 & 61 & 42 & 0,806 \\
\hline Mín./máx. & $14 / 85$ & $29 / 80$ & $14 / 85$ & \\
\hline
\end{tabular}

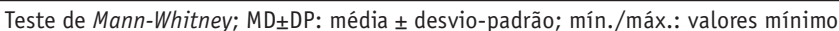
e máximo das variáveis; $p$ : nível descritivo

\section{DISCUSSÃO}

O curso clínico da osteonecrose é usualmente progressivo para o estágio final, com alterações degenerativas secundárias da articulação acometida, destruição articular, dor e limitação de movimentos ${ }^{(2)}$. Em pacientes jovens com LES a osteonecrose tem sido apontada como importante causa de incapacidade física, ocasionando prejuízo da qualidade de vida desses pacientes ${ }^{(15,23)}$.

Osteonecrose foi previamente observada em 4\% a 6\% dos pacientes com LES em dois estudos brasileiros ${ }^{(24,25)}$. Uma das possíveis explicações para as diferenças porcentuais das freqüências desta co-morbidade descritas na literatura seria a variabilidade da amostra de pacientes e a gravidade do LES.

A média de idade de nossos pacientes à época de apresentação da osteonecrose foi 29 anos e a média de idade à época do diagnóstico de LES foi 23 anos, semelhantes aos dados observados por Mok et al. ${ }^{(17)}$ A média do intervalo de tempo entre o diagnóstico de LES e a detecção de osteonecrose foi 6 anos, período menor que o relatado por outros estudos, que verificaram variação de 8 e 9 anos $^{(15,17)}$.

A cabeça femoral foi o sítio de osteonecrose mais acometido, em concordância com outros relatos. A distribuição bilateral de osteonecrose de quadril esteve presente em $77 \%$ dos pacientes, assim como referido na literatura ${ }^{(8,16,17)}$.

Acredita-se que a patogênese da osteonecrose em pacientes com LES seja multifatorial, incluindo-se alguns fatores de risco específicos relacionados à doença. Fenômeno de Raynaud foi associado à osteonecrose em estudo anterior ${ }^{(14)}$, mas não confirmado por outros pesquisadores posteriormente ${ }^{(5,8,9,26)}$. Dos pacientes com osteonecrose estudados, $57 \%$ apresentaram fenômeno de Raynaud, porém, esta frequiência não foi significantemente diferente da observada nos pacientes sem osteonecrose (50\%). Por outro lado, um estudo com ressonância nuclear magnética encontrou associação significante entre fenômeno de Raynaud e osteonecrose assintomática ${ }^{(6)}$.

Estudo anterior relatou que pacientes com osteonecrose apresentaram incidência significantemente maior de doença neurológica quando comparados aos pacientes com LES sem osteonecrose $\mathrm{e}^{(8)}$. Neste estudo, não houve diferença significante com relação à manifestação neuropsiquiátrica pregressa entre os subgrupos de pacientes analisados, em conformidade com o estudo de Gladman et al. ${ }^{(9)}$.

Pacientes com osteonecrose apresentaram maior porcentagem de outros acometimentos musculoesqueléticos permanentes quando comparados aos pacientes sem osteonecrose. Isto poderia ser atribuído à gravidade do LES, que acarreta danos permanentes em vários órgãos e/ou sistemas.

História prévia de vasculite digital foi fator de risco para o desenvolvimento de osteonecrose neste estudo. O risco de pacientes com vasculite digital pregressa apresentar osteonecrose foi nove vezes maior que o de pacientes com LES sem vasculite prévia. Não observamos associação significante entre outras manifestações vasculares (trombose venosa, vasculite de mucosas ou de vasos maiores) e osteonecrose. Mont et al. sugeriram que pacientes com evidências clínicas de trombose venosa ou vasculite sofreriam maior risco de desenvolver osteonecrose. Neste estudo, os pacientes com osteonecrose também diferiram daqueles sem osteonecrose quanto ao hábito de fumo e presença de préeclâmpsia ${ }^{(7)}$.

Outro possível fator de risco para osteonecrose em LES seria a presença de anticorpos antifosfolípides. Anticorpos anticardiolipina da classe $\operatorname{IgG}^{(7)}$ e anticoagulante lúpico ${ }^{(8)}$ foram variáveis descritas em associação com osteonecrose. Sheikh et al. sugeriram associação entre piora da fibrinólise e osteonecrose em LES ${ }^{(18)}$. Não observamos associação entre anticorpos anticardiolipina e osteonecrose, em concordância com estudos que demonstraram prevalência similar de títulos de anticorpos antifosfolípides entre pacientes com e sem osteonecrose ${ }^{(11,17,26)}$.

A associação entre corticoterapia e osteonecrose é bem estabelecida ${ }^{(2)}$. Os esteróides fazem parte do arsenal tera- 
pêutico do LES, sendo administrados de acordo com a gravidade da doença e o tipo de envolvimento orgânico. É difícil determinar o grau de implicação da corticoterapia per se ou da gravidade do LES no desenvolvimento da osteonecrose, uma vez que pacientes com doença mais ativa e mais grave são tratados com doses maiores de corticosteróides e por período de uso mais prolongado.

Todos os pacientes deste estudo, em algum momento ao longo da doença, receberam esteróides. Não foram observadas associação estatisticamente significante entre tempo de uso ou dose cumulativa de prednisona e presença de osteonecrose na pequena amostra analisada. Zizic et al. ${ }^{(14)}$ e Mok et al. ${ }^{(17)}$ também não demonstraram diferença quanto à dose cumulativa de corticosteróides na avaliação dos subgrupos de pacientes com e sem osteonecrose. Foram incluídos nas análises estatísticas apenas os pacientes regularmente acompanhados na instituição, visando a minimizar erros de cálculo nas consultas dos prontuários médicos. Assim, embora com reduzido número de pacientes, observa-se que nem todos aqueles que receberam altas doses de esteróides desenvolveram osteonecrose. $\mathrm{Na}$ ausência da correlação com dose total cumulativa de esteróides é possível que a osteonecrose reflita a sensibilidade específica de cada paciente ao corticosteróide.

Neste estudo, a média da dose cumulativa de prednisona dos pacientes que desenvolveram osteonecrose foi $58.000 \mathrm{mg}$, valor superior ao estimado por outros autores $^{(14,26)}$. A média da dose cumulativa de prednisona do grupo controle estudado $(49.000 \mathrm{mg})$ também foi superior à observada em pacientes com LES sem osteonecrose ${ }^{(14)}$. Rascu et al. analisaram 280 pacientes com LES acompanhados por dez anos e observaram menor dose cumulativa de esteróides em pacientes que desenvolveram osteonecrose $(38.834 \mathrm{mg})^{(26)}$, quando este dado foi comparado ao estudo de Zizic et al. $(45.300 \mathrm{mg})^{(14)}$.

Por outro lado, maior duração de esteróides em período de anos, maior dose cumulativa e maior dose máxima de corticosteróides foram as variáveis associadas com osteonecrose no estudo de 95 pacientes ${ }^{(9)}$. Maior dose diária de prednisona foi correlacionada com a osteonecrose, a adição de sítios ósseos e o número de locais acometidos em estudo clínico com 31 pacientes $_{(7)}$. O início da osteonecrose ocorreu durante o primeiro mês de tratamento com altas doses de corticosteróides ${ }^{(27)}$. Outro estudo, com 38 pacientes com LES e osteonecrose, revelou que maior dose cumulativa de esteróides administrada nos quatro primeiros meses de diagnóstico de LES foi fator de risco independente para osteonecrose $^{(8)}$.
Corticoterapia correlacionou-se fortemente com gravidade de osteonecrose. Pacientes com doença óssea mais grave (igual ou superior a 6 sítios de osteonecrose) receberam maior dose diária de prednisolona do que pacientes com lesão óssea menos grave (1 ou 2 sítios de osteonecrose $)^{(11)}$. Neste estudo, apenas um paciente apresentou 6 sítios de osteonecrose. Do total de pacientes, 79\% sofreram comprometimento de 1 ou 2 sítios ósseos, sendo que $50 \%$ recebiam pelo menos $20 \mathrm{mg}$ diários de prednisona à época da detecção da osteonecrose.

Adicionalmente, certas alterações corpóreas habituais observadas em resposta à corticoterapia, como Cushing, moon facies, giba de búfalo, obesidade no tronco e osteopenia foram associadas com osteonecrose em dois estudos ${ }^{(7,8)}$.

Além dos fatores de risco relacionados com o início da osteonecrose, aspectos implicados com o aumento do risco de progressão clínica da doença devem ser compreendidos. Nesse sentido, a localização da lesão óssea, o tamanho radiográfico da lesão e o estágio radiográfico da doença devem ser considerados. Mont et al. estudaram os fatores de risco envolvidos na progressão da osteonecrose associada com corticosteróides nos quadris de 50 pacientes (18 destes com LES) submetidos à descompressão medular. A maioria dos quadris dos pacientes com LES apresentaram-se com doença pós-colapsal, evoluindo precariamente após o tratamento baseado na descompressão medular. Estágio tardio de osteonecrose na apresentação e extensão radiológica da lesão foram preditores de progressão da osteonecrose ${ }^{(28)}$.

Ressalta-se que, neste estudo, a definição dos grupos de pacientes com osteonecrose e controle foi baseada em dados obtidos através de prontuários médicos. Dessa forma, pacientes com osteonecrose pouco sintomática ou assintomática podem ter sido excluídos e a casuística em questão representar uma seleção de casos mais complicados e/ou em estágio mais avançado da doença. Os pacientes do grupo considerado controle não foram submetidos à avaliação diagnóstica para a detecção de osteonecrose, sendo alocados nesse grupo apenas por não haver dados de prontuários que sugerissem a presença desta co-morbidade.

Este estudo demonstra a relevância da vasculite digital como dado clínico associado à osteonecrose em LES, levantando a possibilidade de a vasculite per se ser a causa da osteonecrose observada nesses pacientes. Enfatiza a necessidade de se proceder à avaliação de todos os possíveis fatores de risco inerentes ou associados à osteonecrose como complicação relacionada ao $\operatorname{LES}^{(7,8,14,18,19,29,30)}$, a definição do diagnóstico precoce do evento ósseo isquêmico através de radiografia simples ${ }^{(31)}$ ou de ressonância nuclear magné- 
tica $^{(32,33)}$ e o tratamento adequado $^{(34)}$, a fim de se reduzir a progressão desta co-morbidade ${ }^{(23)}$.

Deve-se evitar o uso de altas doses de corticosteróides em pacientes com alguma articulação comprometida, a fim de proteger os outros sítios ósseos da ação deletéria da corticoterapia. Para tanto, orienta-se o uso de agentes poupadores de corticosteróides como as drogas antimaláricas e azatioprina.

\section{REFERÊNCIAS}

1. Sweet DE, Madewell JE: Osteonecrosis - Pathogenesis. In: Resnick D (ed.). Diagnosis of bone and joint disorders. 3rd ed., United States: WB Saunders Company, 1995 (section XV, chapter 79 p. 3445-94).

2. Assouline-Dayan Y, Chang C, Greenspan A, Shoenfeld Y, Gershwin ME: Pathogenesis and natural history of osteonecrosis. Semin Arthritis Rheum 32: 94-124, 2002.

3. Dubois EL, Cozen L: Avascular (aseptic) bone necrosis associated with systemic lupus erythematosus. JAMA 174: 966-71, 1960.

4. Gladman DD, Urowitz MB: Morbidity in systemic lupus erythematosus. J Rheumatol 14 (suppl 13): 223-6, 1987.

5. Weiner ES, Abeles M: Aseptic necrosis and glucocorticosteroids in systemic lupus erythematosus - a reevaluation. J Rheumatol 16 604-8, 1989.

6. Aranow C, Zelicof S, Leslie D, et al: Clinically occult avascular necrosis of the hip in systemic lupus erythematosus. J Rheumatol 24: 2318-22, 1997

7. Mont MA, Glueck CJ, Pacheco IH, Wang P, Hungerford DS, Petri M: Risk factors for osteonecrosis in systemic lupus erythematosus. J Rheumatol 24: 654-62, 1997.

8. Mok CC, Lau CS, Wong RWS: Risk factors for avascular bone necrosis in systemic lupus erythematosus. Br J Rheumatol 37: 895-900, 1998

9. Gladman DD, Urowitz MB, Chaudhry-Ahluwalia V, Hallet DC, Cook RJ: Predictive factors for symptomatic osteonecrosis in patients with systemic lupus erythematosus. J Rheumatol 28: 761-5, 2001.

10. Halland AM, Klemp P, Botes D, Van Heerden BB, Loxton A, Scher AT: Avascular necrosis of the hip in systemic lupus erythematosus the role of magnetic resonance imaging. Br J Rheumatol 32: 972-6, 1993.

11. Houssiau FA, Toukap AN, Depresseux G, et al: Magnetic resonance imaging-detected avascular osteonecrosis in systemic lupus erythematosus - lack of correlation with antiphospholipid antibodies. Br J Rheumatol 37: 448-53, 1998.

12. Gladman D, Ginzler E, Goldsmith C, et al: The development and initial validation of the systemic lupus international collaborating clinics/American College of Rheumatology damage index for systemic lupus erythematosus. Arthritis Rheum 39: 363-9, 1996.

13. Gladman DD, Urowitz MB, Goldsmith $\mathrm{CH}$, et al: The reliability of the systemic lupus international collaborating clinics/American College of Rheumatology damage index in patients with systemic lupus erythematosus. Arthritis Rheum 40: 809-13, 1997.

14. Zizic TM, Marcoux C, Hungerford DS, Dansereau JV, Stevens MB: Corticosteroid therapy associated with ischemic necrosis of bone in systemic lupus erythematosus. Am J Med 79: 596-604, 1985.
Há, na literatura, relatos de bons a excelentes resultados clínicos após artroplastia total de quadril em pacientes com $\mathrm{LES}^{(35,36)}$. Quatro dos pacientes estudados foram submetidos à cirurgia para a colocação de próteses de quadril (unilateral em dois casos e bilateral em outros dois casos). Os demais pacientes se mantêm sob tratamento conservador em reabilitação física.

15. Gladman DD, Chaudhry-Ahluwalia V, Ibañez D, Bogoch E, Urowitz MB: Outcomes of symptomatic osteonecrosis in 95 patients with systemic lupus erythematosus. J Rheumatology 28: 2226-9, 2001.

16. Klippel JH, Gerber LH, Pollak L, Deker JL: Avascular necrosis in systemic lupus erythematosus. Am J Med 67: 83-7, 1979.

17. Mok MY, Farewell VT, Isenberg DA: Risk factors for avascular necrosis of bone in patients with systemic lupus erythematosus - is there a role for antiphospholipid antibodies? Ann Rheum Dis 59: 462-7, 2000

18. Sheikh JS, Retzinger GS, Hess EV: Association of osteonecrosis in systemic lupus erythematosus with abnormalities of fibrinolysis. Lupus 7: 42-8, 1998

19. Jones LC, Mont MA, Le TB, et al: Procoagulants and osteonecrosis. J Rheumatology 30: 783-91, 2003.

20. Tan EM, Cohen AS, Fries JF, et al: The 1982 revised criteria for the classification of systemic lupus erythematosus. Arthritis Rheum 25: 1271-7, 1982.

21. Hochberg MC: Updating the American College of Rheumatology revised criteria for the classification of systemic lupus erythematosus. Arthritis Rheum 40: 1725, 1997 (letter).

22. Siegel J: Estatística não-paramétrica, Brasil, McGraw Hill do Brasil, 1981, 350p.

23. Urowitz MB, Gladman DD: How to improve morbidity and mortality in systemic lupus erythematosus. Rheumatology 39 238-44, 2000

24. Sella EMC, Sato EI: Avaliação do índice de danos permanentes através de SLICC/ACR-DI em pacientes com mais de cinco anos de diagnóstico de lúpus eritematoso sistêmico. Rev Bras Reumatol 44: 115-22, 2004.

25. Costallat LTL, Coimbra AMV: Lúpus eritematoso sistêmico - análise clínica e laboratorial de 272 pacientes em um hospital universitário (1973-1992). Rev Bras Reumatol 35: 23-9, 1995.

26. Rascu A, Manger K, Kraetsch HG, Kalden JR, Manger B: Osteonecrosis in systemic lupus erythematosus, steroid-induced or a lupus-dependent manifestation? Lupus 5: 323-7, 1996.

27. Oinuma K, Harada Y, Nawata Y, et al: Osteonecrosis in patients with systemic lupus erythematosus develops very early after starting high dose corticosteroid treatment. Ann Rheum Dis 60: 1145-8, 2001.

28. Mont MA, Fairbank AC, Petri M, Hungerford DS: Core decompression for osteonecrosis of the femoral head in systemic lupus erythematosus. Clin Orthop 334: 91-7, 1997

29. Maxwell SRJ, Moots RJ, Kendall MJ: Corticosteroids - do they damage the cardiovascular system? Postgrad Med 70: 863-70, 1994.

30. American College of Rheumatology Ad Hoc Committee on systemic lupus erythematosus guidelines. Guidelines for referral and management of systemic lupus erythematosus in adults. Arthritis Rheum 42: 1785-96, 1999. 
31. Chaudhry-Ahlawalia V, Gladman DD, Urowitz MB, Bogoch E, Farewell VT: Radiographic reports in osteonecrosis of the hip in systemic lupus erythematosus. Clin Orthop 352: 131-6, 1998.

32. Nagasawa $\mathrm{K}$, Tsukamoto $\mathrm{H}$, Tada $\mathrm{Y}$, et al: Imaging study on the mode of development and changes in avascular necrosis of the femoral head in systemic lupus erythematosus - long-term observations. Br J Rheum 33: 343-7, 1994.

33. Sugano N, Ohzono K, Masuhara K, Takaoka K, Ono K: Prognostication of osteonecrosis in the femoral head in patients with systemic lupus erythematosus by magnetic resonance imaging. Clin Orthop 305: 190-9, 1994
34. Mont MA, Jones LC: Management of osteonecrosis in systemic lupus erythematosus. Rheum Dis Clin North Am 26: 279-309, 2000.

35. Hanssen AD, Cabanela ME, Michet Jr CJ: Hip arthroplasty in patients with systemic lupus erythematosus. J Bone and Joint Surg 69-A: 807-14, 1987.

36. Zangger P, Gladman DD, Urowitz MB, Bogoch ER: Outcome of total hip replacement for avascular necrosis in systemic lupus erythematosus. J Rheumatol 27: 919-23, 2000. 